\title{
Unitarity Constraints on Semi-hard Jet Production in Impact Parameter Space
}

\author{
T. C. Rogers \\ Department of Physics, Pennsylvania State University, \\ University Park, PA 16802, USA \\ A. M. Staśto \\ Department of Physics, Pennsylvania State University, \\ University Park, PA 16802, USA and \\ H. Niewodniczański Institute of Nuclear Physics, Kraków, Poland \\ M. I. Strikman \\ Department of Physics, Pennsylvania State University, \\ University Park, PA 16802, USA
}

\begin{abstract}
The perturbative QCD formula for minijet production consitutes an important ingredient in models describing the total cross section and multiparticle production in hadron-hadron scattering at high energies. Using arguments based on s-channel unitarity we set bounds on the minimum value of $p_{t}$ for which the leading twist minijet formula can be used. For large impact parameters where correlations between partons appear to be small we find that the minimum value of $p_{t}^{c}$ should be greater than $2.5 \mathrm{GeV}$ for $\mathrm{LHC}$ energies and greater than $3.5 \mathrm{GeV}$ for cosmic ray energies of about $50 \mathrm{TeV}$. We also argue that for collisions with values of impact parameters typical for heavy particle production the values of minimum $p_{t}$ are likely to be considerably larger. We also analyze and quantify the potential role of saturation effects in the gluon density. We find that although saturation effects alone are not sufficient to restore unitarity, they are likely to play an important role at LHC energies.
\end{abstract}

Keywords: perturbative QCD, minijet production, impact parameter, unitarity, gluon saturation

\section{INTRODUCTION}

The high energy hadronic interactions which will be studied at the LHC, and the interaction of ultra-high energy cosmic rays in the atmosphere both involve QCD effects that are still poorly understood and largely unexplored experimentally. Therefore, in extrapolating Monte Carlo simulations to these very high energies, one often must resort to phenomenological models [1, 2, 3, 4, 5, 6] based on fits to lower energy data. Of particular interest is the contribution from jets with moderate relative transverse momentum $p_{t}$ (so-called minijets or semi-hard jets).

The basic input to these models is the total inclusive minijet cross section in hadron-hadron scattering, typically calculated using the leading-twist perturbative QCD (pQCD) factorization formula. Schematically, the minijet cross section is the convolution integral of a parton density for each colliding hadron and a partonic cross section for two-to-two parton scattering,

$$
\sigma_{2 j e t}^{i n c} \sim \int_{p_{t}^{c}}^{\infty} d p_{t}^{2} \frac{d \hat{\sigma}}{d p_{t}^{2}} f_{h_{1}}\left(x_{1}, \mu^{2}\right) \otimes f_{h_{2}}\left(x_{2}, \mu^{2}\right),
$$

where $\otimes$ denotes convolution integrals in longitudinal momentum fractions $x_{1}, x_{2}$ for each of the colliding hadrons. The scale $\mu$ is the factorization scale which is usually set to be equal to $p_{t}$, the transverse momentum. Equation (11) has to be regulated in the low momentum regime by introducing a cutoff $p_{t}^{c}$. Equation (1) constitutes the semi-hard part in most models of high energy hadron scattering. The part which includes soft interactions must be modeled separately.

Since the integrand in Eq. (11) is sharply peaked at very low transverse momenta, the minijet cross section is highly sensitive to the value of the cutoff $p_{t}^{c}$. The problem of determining a minimum $p_{t}$ is generic to all the models which aim to describe the particle production in hadron collisions [1, 2, 3, 3, 4, 5, 6, 7, 8].

The true range of validity for $p_{t}^{c}$ remains a point of some controversy. The leading twist pQCD expression for Eq. (1) is most reliable when $p_{t}^{c}$ is large. However, one hopes to utilize the full power of pQCD for as wide a range of kinematics as possible.

Imposing unitarity in impact parameter space provides a potential constraint on allowed values of $p_{t}^{c}$ at very high energies. Unlike in the case of the total cross section, however, the unitarity constraints cannot be imposed directly on (10), which is an inclusive cross section proportional to average jet multiplicity. Therefore, less direct methods which take jet multiplicity into account are needed. A parton model based picture of multiple interactions has been shown to be a natural framework [9] with which to address the unitarity issue. This approach has been applied, for example, in [10] in the context of Monte Carlos for cosmic ray air showers.

In this paper, we propose a simple method for constraining the minimum value of $p_{t}^{c}$ at large impact parameters $(b \gtrsim 1.5 \mathrm{fm})$ for which the minijet formula in Eq. (1) makes sense. Our method is based on s-channel unitarity of the amplitude in the impact parameter representation. Our results are sensitive only to the transverse 
distribution of gluons in the nucleon which is measured in small- $x$ hard exclusive processes. We produce conservative bounds on $p_{t}^{c}$ - the true minimum $p_{t}^{c}$ should be higher than what we find. In this paper we restrict attention to large impact parameters where it is reasonable to neglect parton correlations. Apart from this, however, we avoid discussing any specific model of the underlying dynamics responsible for unitarization. Nevertheless, the approach we use in this paper is likely to be applicable at smaller impact parameters, given a particular model of parton correlations.

The lower bound we obtain on the transverse momentum cutoff is at least $2.5 \mathrm{GeV}$ for center-of-mass pp collisions at $14 \mathrm{TeV}$ and impact parameters $\gtrsim 1.5 \mathrm{fm}$. We also consider here much higher energies, $\sqrt{s} \approx 50 \mathrm{TeV}$ which are relevant to cosmic ray studies. Here, we find that the minimum cutoff probably becomes closer to $3.5 \mathrm{GeV}$ for the same values of impact parameters for $p p$ collisions. We also discuss possibilities for extending the analysis to smaller impact parameters.

The structure of the paper is as follows: in the next section we introduce the formula for minijet production. By investigating the integrand at fixed values of $p_{t}$ and the energy $\sqrt{s}$ we constrain the range in the rapidities and $x$ values that dominates the integral. On the basis of the saturation scale from the Golec-Biernat and Wusthoff model (GBW) we estimate the role of the saturation corrections in the gluon density at the Tevatron and at the LHC.

In Sec. III we develop a general method based on unitarity arguments for constraining the minimum value of the $p_{t}$ for the minijet formula to be used.

In Sec. IV we give a general discussion of the radius of the interaction in the context of the eikonal model. We make comparisons here to the interaction radius for deep inelastic scattering (DIS)

Finally, in the appendix we include the formulae for the dependence of the mass parameter on $x$ and the scale in the profile function.

\section{MINIJET CROSS SECTION}

We begin by writing Eq. (1) more explicitly. The inclusive minijet cross section in hadron-hadron scattering is typically calculated using the leading-twist perturbative QCD (pQCD) factorization formula,

$$
\begin{array}{r}
\sigma_{2 j e t}^{i n c}\left(s, p_{t}^{\mathrm{c}}\right)=\sum_{i, j, k, l} \frac{K}{1+\delta_{k l}} \int d x_{1} d x_{2} \int d p_{t}^{2} \times \\
\times \frac{d \hat{\sigma}_{i j \rightarrow k l}}{d p_{t}^{2}} f_{i}\left(x_{1}, \mu^{2}\right) f_{j}\left(x_{2}, \mu^{2}\right) \theta\left(p_{t}-p_{t}^{c}\right) .
\end{array}
$$

Here, the differential cross section $d \hat{\sigma} / d p_{t}^{2}$ is for two-to-two parton scattering. In this work we calculate it at the lowest order in pQCD. The standard leading twist parton distribution function, $f_{j}\left(x, \mu^{2}\right)$ is evaluated at a hard scale $\mu^{2}$ which is typically chosen to be the transverse momentum squared, $p_{t}^{2}$. The factor $1 /\left(1+\delta_{k l}\right)$ takes into account necessary symmetry factors when the outgoing partons are identical. The $K$ is a factor used to correct for higher order terms, and is fixed to 2 in many models. In NLO treatments of single jets, $K$ is found to be closer to 1.0. However since processes where at least three jets are produced appear to be dominant at these energies $K=1$ corresponds to effective $K \sim 1.5$ for our observable. In keeping with our goal of maintaining conservative estimates of unitarity bounds, we take this value for the $K$ factor.

The kinematic limit for the $x_{1}, x_{2}$ integrations is

$$
x_{1} x_{2} s>4\left(p_{t}^{c}\right)^{2} .
$$

As we will see shortly, in the UHE limit and at small $p_{t}$, the dominant range of the integration in (2) is over small values of $x$ where the gluon density, $g\left(x, p_{t}^{2}\right)$ dominates. A continuing source of uncertainty is the lowest value of the $p_{T}^{c}$ for which Eq. (2) makes sense. Since the cutoff is in the regime where the integrand begins to spike, small changes in the cutoff can lead to dramatic changes in the integrated cross section, Eq. (2). This is illustrated in Fig. 1 where the integrated minijet cross section is shown as a function of the c.m.s. energy for different values of the $p_{t}^{\mathrm{c}}$. We see a drastic change in the normalization, although the shape and the rise of the cross section with the energy is universal. This sensitivity to $p_{t}^{c}$ is a symptom of our limited understanding of physics in transitional region between small values of $p_{t}$, where the distribution in transverse momentum must be modeled, and large values where Eq. (2) is a good approximation.

\section{A. The Kinematic Range of Validity for the Minijet Formula}

In order to gain an intuition about the structure of the minijet cross-section at very high energies, it is useful to study the integrand of Eq. (2) as a function of the rapidities $y_{1}, y_{2}$ of the jets. We show a contour plot in Fig. 1 for the integrand versus $y_{1}$ and $y_{2}$, related to $x_{1}$ and $x_{2}$ via the equation,

$$
x_{1,2}=\frac{p_{t}}{\sqrt{s}}\left(e^{ \pm y_{1}}+e^{ \pm y_{2}}\right) .
$$

We use CTEQ6M [18] parton distribution sets for evaluation of Eq. (2). The smallest contours represent the maxima of the integrand. To get an idea of the important regions of the integrand, we have drawn solid thick-red curves along the contours marking half of the peak value. Plots (a) and (b) show the result of calculations done at $1.96 \mathrm{TeV}$ and $14 \mathrm{TeV}$ respectively. In both cases, $p_{t}$ is fixed at $2.5 \mathrm{GeV}$. Note that as $\sqrt{s}$ increases, the peaks begin to form long ridges that extend toward the lowerleft and upper-right corners of the plots where $y_{1}$ and $y_{2}$ have the same sign. The ridges also expand if we decrease 
$p_{t}$, as shown in plot (c) where $p_{t}=1.5 \mathrm{GeV}$, still with $\sqrt{s}=14 \mathrm{TeV}$.

For figure 2(a) the peaks are located at rapidities,

$$
y_{1} \simeq-y_{2} \simeq \pm 1.2,
$$

and so the $x$ values probed in the collision with this configuration are approximately equal $x_{1} \simeq x_{2} \simeq 0.005$. Still, at rapidities

$$
\left|y_{1}\right| \simeq 4,\left|y_{2}\right| \simeq 2, \text { and } \operatorname{sign} y_{1}=\operatorname{sign} y_{2},
$$

the integrand is rather large. Such a configuration corresponds to asymmetric values of $x_{1}, x_{2}$,

$$
x_{1,2} \simeq 2 \times 10^{-4} \ll x_{2,1} \simeq 0.08 .
$$

At the maximum LHC energy - $14 \mathrm{TeV}$ - plotted in figure (b), the central values are, $\Longrightarrow x_{1} \leftrightarrow x_{2}$

$$
x_{1} \simeq x_{2} \simeq 0.0008,
$$

but the ridges extend toward values of rapidity,

$$
\left|y_{1}\right| \simeq 6,\left|y_{2}\right| \simeq 4
$$

corresponding to $x_{1,2} \simeq 4 \times 10^{-6} \ll x_{2,1} \simeq 0.08$. Therefore, both aligned and anti-aligned jets become important.

In the regions where $y_{1}$ and $y_{2}$ are both very large and of the same sign, one of the values of $x$ becomes very small. The is the regime where one of the gluon densities begins to blow up, and one expects non-linear effects (saturation) to come into play, see for example [11], 112]. For a given value of $x$ saturation models predict that $g(x, \mu)$ becomes saturated for values of $\mu$ below the saturation scale, $Q_{s}$. Studies of deep inelastic scattering using various versions of the $\mathrm{pQCD}$ dipole picture [13, 14, 15, 16, 17] have resulted in rough bounds on where saturation effects should begin to become significant. To illustrate the sensitivity of the integrand to the saturation effects in the gluon density we superimpose the saturation scale from the GBW model [13], 14] onto our contour plots. The blue lines in the corners of the contour figures correspond to the saturation line 24] from the GBW model. These lines mark off the regions of saturation in the upper right-hand and lower left-hand corners of the figures. Values of $y_{1}, y_{2}$ that lie within the blocks marked off by the saturation line indicate regions where either $g\left(x_{1}, p_{t}\right)$ or $g\left(x_{2}, p_{t}\right)$ is likely to be very sensitive to saturation effects since either $Q_{s}\left(x_{1}\right)$ or $Q_{s}\left(x_{2}\right)$ are larger than the fixed value of $p_{t}$. The percentage of the integral affected by the saturation corrections is about $\sim 11 \%$ for the $p_{t}=2.5 \mathrm{GeV}$ and $\sim 53 \%$ for $p_{t}=1.5 \mathrm{GeV}$ for the LHC energy. We did not show the saturation lines for the Tevatron energy since for this choice of $p_{t}=2.5 \mathrm{GeV}$ the saturation effects are very small. We note, however, that the model used in [16] allows $x_{1}, x_{2}$ to go to somewhat smaller values without actually violating unitarity.

When evaluating the saturation effects one has to take into account the difference between the saturation scale

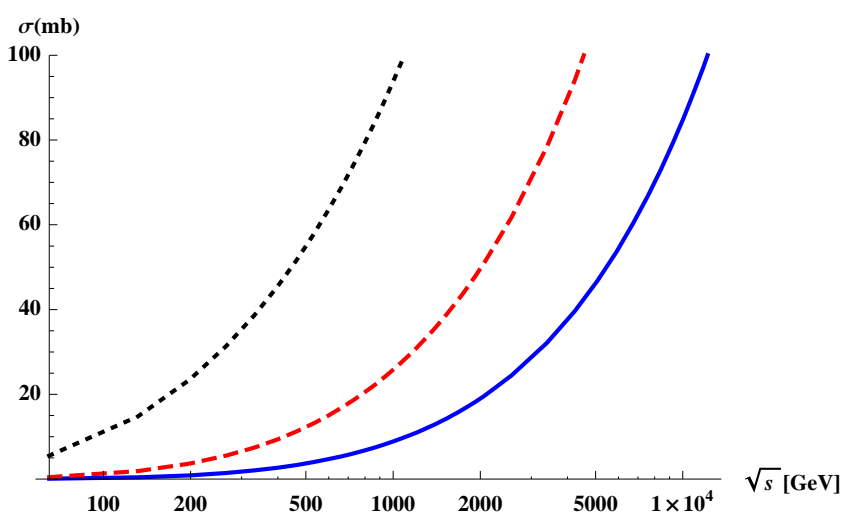

FIG. 1: Inclusive minijet cross section evaluated from (2) for three different cutoff values $p_{t}^{c}=1.5$ (dotted line), $p_{t}^{c}=2.5$ (dashed line) and $p_{t}^{c}=3.5 \mathrm{GeV}$ (solid line). The $K$ factor is set to one in the plots. Parton distributions are CTEQ6M.

for the quark and gluon dipoles. Since here the dominant process is $g g \rightarrow g g$ scattering the saturation scale is enhanced by an additional color factor $C_{A} / C_{F}=9 / 4$ relative [19] to the case for the quark scattering. We therefore include the corresponding rescaled saturation region in Fig. 2, marked off by the solid blue line (as opposed to the dashed-blue which corresponds to the original unrescaled value of the saturation scale from the GBW model).

Furthermore, our analysis has only considered the leading $g g \rightarrow g g$ contribution so far. In the calculation of the total inclusive minijet cross section we include contributions from all channels. The $g g \rightarrow g g$ channel is of course dominant, it is about $60-70 \%$ of the total value depending on the choice of the parton density and the c.m.s. energy. The $q g \rightarrow q g$ contribution however, is non-negligible and constitutes about $30 \%$ of the minijet cross section. It is particularly important for asymmetric configurations of the minijets. In this case the large contribution comes from the region where the quark density is evaluated at large $x$ and the gluon density is evaluated at very small values of $x$.

Figures 2(a-b) demonstrate that gluon saturation effects will likely be significant at LHC energies and higher. However, we stress that other effects (e.g. multiple scattering) are equally important for unitarizing the $p p$ cross section. In fact the multiple hadron scattering effects are likely to become dominant at the lower energies, in a regime where the saturation of the gluon density is not very large. In this paper, we take a very general approach that does not distinguish the underlying physics responsible for unitarization.

\section{IMPACT PARAMETER DEPENDENCE OF THE MINIJET CROSS SECTION}

In this section we review the basic elements needed to discuss unitarity in impact parameter space. 


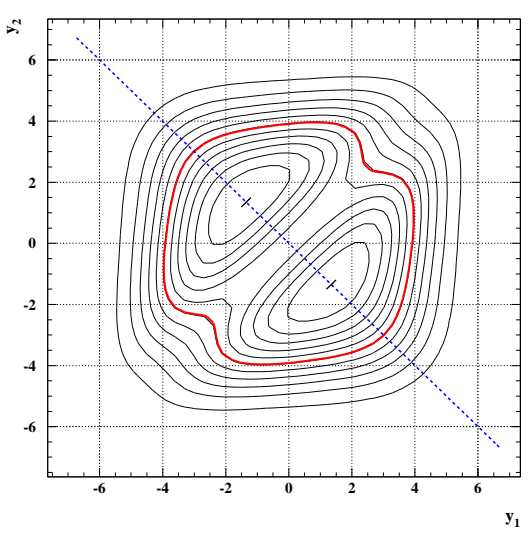

(a)

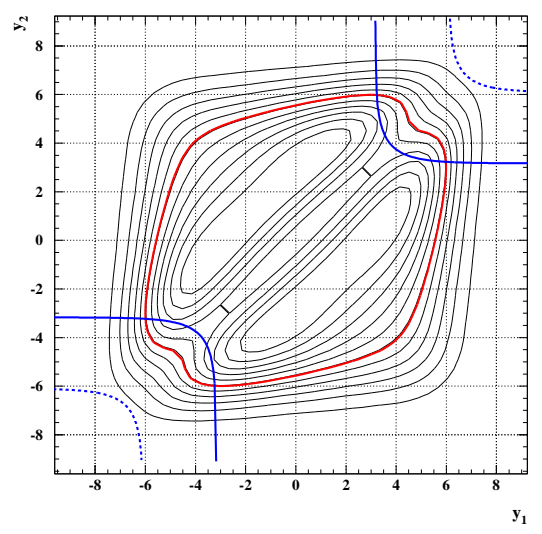

(b)

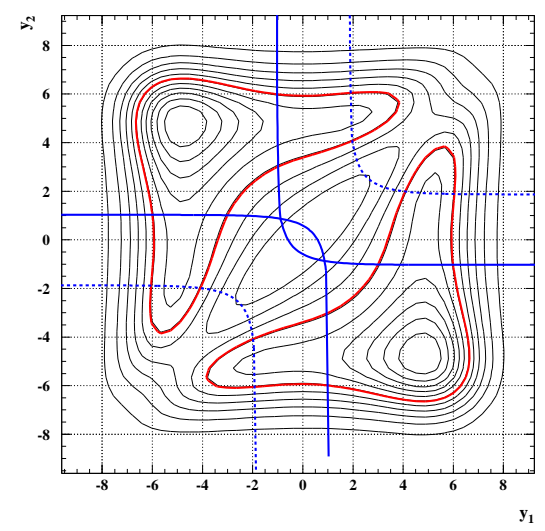

(c)

FIG. 2: Rapidity distribution of the integrand in the minijet cross section formula for (a) $\sqrt{s}=1.96 \mathrm{TeV}, p_{t}=2.5 \mathrm{GeV},(\mathrm{b})$ $\sqrt{s}=14 \mathrm{TeV}, p_{t}=2.5 \mathrm{GeV}$ and (c) $\sqrt{s}=14 \mathrm{TeV}, p_{t}=1.5 \mathrm{GeV}$. The calculation is done for the gluon-gluon channel only. The thick - red contour is where the integrand is $50 \%$ of the maximum value. The corner regions marked off by the dotted blue lines indicate where saturation effects may be present according to the GBW model. The solid blue line is the result of rescaling the saturation scale by a factor of $9 / 4$ as discussed in the text.

\section{A. Basic Formulation}

Following standard steps the total, elastic and inelastic cross sections can be written at high energies as,

$$
\begin{aligned}
\sigma_{\text {tot }}(s) & =2 \int d^{2} \mathbf{b} \operatorname{Re} \Gamma(s, b), \\
\sigma_{e l}(s) & =\int d^{2} \mathbf{b}|\Gamma(s, b)|^{2}, \\
\sigma_{\text {inel }}(s) & =\int d^{2} \mathbf{b}\left(2 \operatorname{Re} \Gamma(s, b)-|\Gamma(s, b)|^{2}\right),
\end{aligned}
$$

where the profile function $\Gamma(s, b)$ is the Fourier transform in impact parameter of the scattering amplitude for twoto-two process

$$
\Gamma(s, b)=\frac{1}{2 i s(2 \pi)^{2}} \int d^{2} \mathbf{q} e^{i \mathbf{q} \cdot \mathbf{b}} A(s, t) .
$$

Assuming that the amplitude is dominantly imaginary (which is appropriate at high energies), the unitarity constraint on $\Gamma(b, s)$ is,

$$
\Gamma(s, b) \leq 1
$$

\section{B. Impact Parameter Dependence for Hard Scattering}

We appeal directly to experimental data to obtain the impact parameter dependence of the hard collisions. We use the generalized gluon distribution function extracted directly from $J / \Psi$ electroproduction production [20].

The generalized gluon distribution is related to the standard gluon distribution function and a gluon form factor $F_{g}\left(x, t, \mu^{2}\right)$ through the following defining relation

$$
x f_{g}\left(x, t, \mu^{2}\right)=x f_{g}\left(x, \mu^{2}\right) F_{g}(x, t, \mu),
$$


where

$$
F_{g}(x, t=0, \mu)=1 \text {. }
$$

The Fourier transform of the gluonic form factor gives the profile in impact parameter space

$$
\mathcal{F}_{g}(x, \rho, \mu)=\int d^{2} \boldsymbol{\Delta} F_{g}(x, t, \mu) e^{-i \Delta \cdot \rho}, \quad t=-\Delta^{2},
$$

where the integration over $\rho$ is 2-dimensional. We have the normalization condition

$$
\int d^{2} \rho \mathcal{F}_{g}(x, \rho, \mu)=1,
$$

which is a trivial consequence of the previous condition (9). Interpreting $\mathcal{F}_{g}(x, \rho, \mu)$ as the transverse spatial spread of hard partons, we may write Eq. (2) in the form,

$$
\sigma_{2 j e t}^{i n c}\left(s, p_{t}^{c}\right)=\int d^{2} \mathbf{b} \mathcal{N}_{2}\left(b, s, p_{t}^{c}\right),
$$

where we have defined,

$$
\begin{aligned}
\mathcal{N}_{2}\left(b, s, p_{t}^{c}\right)= & \sum_{k, l} \frac{K}{1+\delta_{k, l}} \times \int_{0}^{1} d x_{1} d x_{2} \int d p_{T}^{2} \times \\
& \times \frac{d \hat{\sigma}_{i j \rightarrow k l}}{d p_{t}^{2}} f_{g}\left(x_{1}, p_{t}^{2}\right) \times \\
& f_{g}\left(x_{2}, p_{t}^{2}\right) P_{2}\left(b, x_{1}, x_{2}, p_{t}\right) \theta\left(p_{t}-p_{t}^{c}\right),
\end{aligned}
$$

with an over-lap function given by,

$$
\begin{aligned}
& P_{2}\left(b, x_{1}, x_{2}, \mu\right)= \\
& \quad \int d^{2} \rho_{\mathbf{1}} \mathcal{F}_{g}\left(x_{1},\left|\rho_{\mathbf{1}}\right|, \mu\right) \mathcal{F}_{g}\left(x_{2},\left|\mathbf{b}-\rho_{\mathbf{1}}\right|, \mu\right) .
\end{aligned}
$$

As usual, we use $\mu=p_{t}$ for the hard scale. It follows from these definitions that $\mathcal{N}_{2}\left(b, s, p_{t}^{c}\right)$ should be interpreted as the relative probability for producing at least one minijet pair at impact parameter $b$ with $p_{t} \geq p_{t}^{c}$ and center of mass energy $\sqrt{s}$.

For a wide range of energies, a reasonable parameterization of the two-gluon form factor is the model of Frankfurt, Strikman, and Weiss (FSW) [21],

$$
F_{g}(x, t, \mu)=\frac{1}{\left(1-\frac{t}{m_{g}^{2}(x, \mu)}\right)^{2}}
$$

Spreading of the form factor at small- $x$ and evolution with the hard scale $\mu$ are taken into account by allowing $m_{g}$ to vary with $x$ and $\mu$. For this, we use the parameterization given in [21] (see Appendix B). Performing the Fourier transform in Eq. (10) while using (13) yields an analytic expression for the impact parameter space gluonic form factor,

$$
\mathcal{F}_{g}(x, \rho, \mu)=\frac{m_{g}^{3}(x, \mu) \rho}{4 \pi} K_{1}\left(m_{g}(x, \mu) \rho\right) .
$$

We make one further approximation to simplify the analysis. The dependence of $m_{g}$ on $x$ is rather slow. Therefore, we make the replacement,

$$
P_{2}\left(b, x_{1}, x_{2}, p_{t}^{c}\right) \longrightarrow P_{2}\left(b, \bar{x}, \bar{x}, p_{t}^{c}\right) \equiv P_{2}\left(b, s, p_{t}^{c}\right),
$$

where $\bar{x} \equiv 2 p_{t}^{c} / \sqrt{s}$. We have calculated $P_{2}\left(b, x_{1}, x_{2}, p_{t}^{c}\right)$ numerically, and have verified that it differs from $P_{2}\left(b, s, p_{t}^{c}\right)$ by no more than ten percent within the essential region of the integrand in Eq. (11). We can then approximate,

$$
\mathcal{N}_{2}\left(b, s, p_{t}^{c}\right) \approx \sigma_{2 j e t}^{i n c}\left(s, p_{t}^{c}\right) P_{2}\left(b, s, p_{t}^{c}\right) .
$$

$P_{2}\left(b, s, p_{t}^{c}\right)$ can then be determined analytically from Eq. (14):

$$
P_{2}\left(b, s, p_{t}^{c}\right)=\frac{m_{g}^{2}\left(\bar{x}, p_{t}^{c}\right)}{12 \pi}\left(\frac{m_{g}\left(\bar{x}, p_{t}^{c}\right) b}{2}\right)^{3} K_{3}\left(m_{g}\left(\bar{x}, p_{t}^{c}\right) b\right)
$$

This quantity is normalized to unity $\int d^{2} b P_{2}(b, s)=1$ and represents the probability density in impact parameter space for the minijet production. Thus, the average squared impact parameter for minijet production is,

$$
\left\langle b^{2}\right\rangle=\int d^{2} \mathbf{b} b^{2} P_{2}\left(b, s, p_{t}^{c}\right) .
$$

This provides a quantitative measure of the width of the overlap function which can then be used to compare with other models.

\section{UNITARITY CONSTRAINTS}

We address the unitarity issue directly by maintaining the impact parameter representation and by calculating, within a particular model, the relative contribution to the total inelastic cross section from the exclusive dijet and $2 k$-jet production cross sections. In this section, we start with a simple model with uncorrelated jet production at large impact parameters.

\section{A. Multiple Collisions}

We follow steps similar to Ref. [9] to take into account multiple collisions. In the simple parton model picture, each collision is a two-to-two collision between partons resulting in an outgoing minijet pair. For production of $k$ minijet pairs, with $k \geq 1$, there is a convolution integral analogous to Eq. (11) which we symbolize by $\mathcal{N}_{2 k}(b, s)$. (Multiple jet production will also involve cutoffs on transverse momentum.) For $k$ minijets, the normalization condition is,

$$
\int d^{2} \mathbf{b} \mathcal{N}_{2 k}(b, s)=\sigma_{2 k}^{i n c}(s),
$$

where $\sigma_{2 k}^{i n c}$ is the $2 k$-jet inclusive cross section. We will refer the $\mathcal{N}_{2 k}(b, s)$ as "impact parameter cross sections" 
since they integrate to total inclusive cross sections for producing $2 k$ minijets. We have not used the symbol $\Gamma(s, b)$ since this is already used to represent the profile function Eq. (4) which integrates to the total cross section. Next, we define the $\tilde{\mathcal{N}}_{2 k}(b, s)(k \geq 1)$ to be the exclusive analogues of the $\tilde{\mathcal{N}}_{2 k}(b, s)$. That is,

$$
\int d^{2} \mathbf{b} \tilde{\mathcal{N}}_{2 k}(b, s)=\sigma_{2 k}^{e x}(s),
$$

is the cross section for producing exactly $k$ minijet pairs at impact parameter $b$. From the definition of exclusive quantities, the inclusive 2-jet impact parameter cross section can be expressed in terms of the exclusive impact parameter cross sections by writing,

$$
\mathcal{N}_{2}\left(b, s, p_{t}^{c}\right)=\sum_{n=1}^{\infty} n \tilde{\mathcal{N}}_{2 n}(b, s) .
$$

More generally, for inclusive $2 k$ jet production we have

$$
\mathcal{N}_{2 k}(b, s)=\sum_{n \geq k}^{\infty}\left(\begin{array}{l}
n \\
k
\end{array}\right) \tilde{\mathcal{N}}_{2 n}(b, s) .
$$

This formula says that to every inclusive $2 k$ jet production process there is a $2 n$ jet exclusive contribution where we have $\left(\begin{array}{l}n \\ k\end{array}\right)$ combination of choosing $k$ pairs from $n$ pairs of jets. Equation (20) can readily be inverted to find the exclusive $\tilde{\mathcal{N}}_{2 k}(b, s)$ in terms of the inclusive $\mathcal{N}_{2 n}(b, s)$,

$$
\tilde{\mathcal{N}}_{2 k}(b, s)=\sum_{n \geq k}^{\infty}\left(\begin{array}{l}
n \\
k
\end{array}\right)(-1)^{n-k} \mathcal{N}_{2 n}(b, s) .
$$

The contribution $\Gamma_{\text {jets }}^{\text {inel }}(b, s)$ from minijet pairs to the total inelastic cross section is by definition the sum over the individual exclusive contributions,

$$
\Gamma_{\text {jets }}^{\text {inel }}(s, b)=\sum_{k=1}^{\infty} \tilde{\mathcal{N}}_{2 k}(b, s) .
$$

Using (21) in (22) produces the simple expression,

$$
\Gamma_{\text {jets }}^{\text {inel }}(s, b)=\sum_{n=1}^{\infty}(-1)^{n-1} \mathcal{N}_{2 n}(b, s) .
$$

$\Gamma_{j e t s}^{i n e l}(s, b)$ would be exactly equal to

$$
\Gamma^{\text {inel }}(s, b)=2 \Gamma(s, b)-|\Gamma(s, b)|^{2},
$$

if minijet production were present in all inelastic events. In general, therefore, we have the unitarity constraint,

$$
\Gamma_{\text {jets }}^{\text {inel }}(s, b) \leq \Gamma^{\text {inel }}(s, b) .
$$

Of course, to evaluate $\Gamma_{\text {jets }}^{\text {inel }}(s, b)$ one needs to know how to calculate the production cross section in impact parameter space for arbitrary number of jets, i.e. one needs a model for each term in Eq. (21).
For this paper, we adopt the simplifying approximations of Eqs. (15, 16) of simplifying (yet reasonable) assumptions. As already mentioned, we consider large impact parameters so that we may neglect correlations. If we interpret $\mathcal{N}_{2}\left(b, \bar{x}, p_{t}^{c}\right)$ as the probability to produce a minijet pair at impact parameter $b$, then we may write the inclusive impact parameter cross section for producing $2 k$ minijet pairs as,

$$
\mathcal{N}_{2 k}(s, b)=\mathcal{N}_{2 k}\left(b, \bar{x}, p_{t}^{c}\right)=\left(\sigma_{2 j e t}^{\text {inc }} P_{2}\left(b, \bar{x}, p_{t}^{c}\right)\right)^{k} .
$$

The expression (23) for a given value of impact parameter is then a geometric series that yields the analytic expression,

$$
\Gamma_{\text {jets }}^{\text {inel }}(s, b)=\sum_{n=1}^{\infty}(-1)^{n-1} \mathcal{N}_{2}^{n}=\frac{\sigma_{2 j e t}^{\text {inc }} P_{2}\left(b, \bar{x}, p_{t}^{c}\right)}{1+\sigma_{2 j e t}^{\text {inc }} P_{2}\left(b, \bar{x}, p_{t}^{c}\right)} .
$$

If the final state partons are identical, then each term in Eq. (24) should carry a symmetry factor of $1 / k$ !. In that case the series in Eq. (25) becomes,

$$
\Gamma_{\text {jets }}^{\text {inel }}(s, b)=1-\exp \left[-\sigma_{2 j e t}^{\text {inc }} P_{2}\left(b, \bar{x}, p_{t}^{c}\right)\right] .
$$

We will investigate both of these cases. Note that, as discussed in [9], Eqs.(25) and (26) satisfy the unitarity condition

$$
\Gamma_{\text {jets }}^{\text {inel }}(s, b) \leq 1,
$$

by construction. Equations (25) and (26) are a reasonable approximation at values of $b$ where $N_{4}(b, s) / N_{2}(b, s)$ is a small parameter so that corrections due to correlations are small. It turns out that this is true for $b \approx 2 \mathrm{fm}$ for energies up to about $\sqrt{s} \approx 50 \mathrm{TeV}$.

In the specific case of four jets the profile function is defined as

$$
P_{4}(b)=\frac{P_{2}(b)^{2}}{\int d^{2} b P_{2}(b)^{2}},
$$

where we have suppressed the other arguments in the profile functions. Note that $P_{4}$ satisfies normalization condition by construction. Likewise the cross section for the four jet production is given by

$$
\sigma_{4 j e t}=\sigma_{2 j e t}^{2} \int d^{2} b P_{2}(b)^{2} .
$$

\section{B. Numerical Calculations}

Figure 3 shows sample calculations using Eq. (25) for the case of (a): $\sqrt{s}=14 \mathrm{TeV}$, relevant to the LHC, and for (b): $\sqrt{s}=50 \mathrm{TeV}$, relevant to cosmic ray air showers. Here we assume that the partons are identical particles. To test the sensitivity to the type of parton distribution being used, we have repreated the calculation using MRST parton distributions in plots $(\mathrm{c}, \mathrm{d})$. We have done the calculation using two values of $p_{t}$ in each 


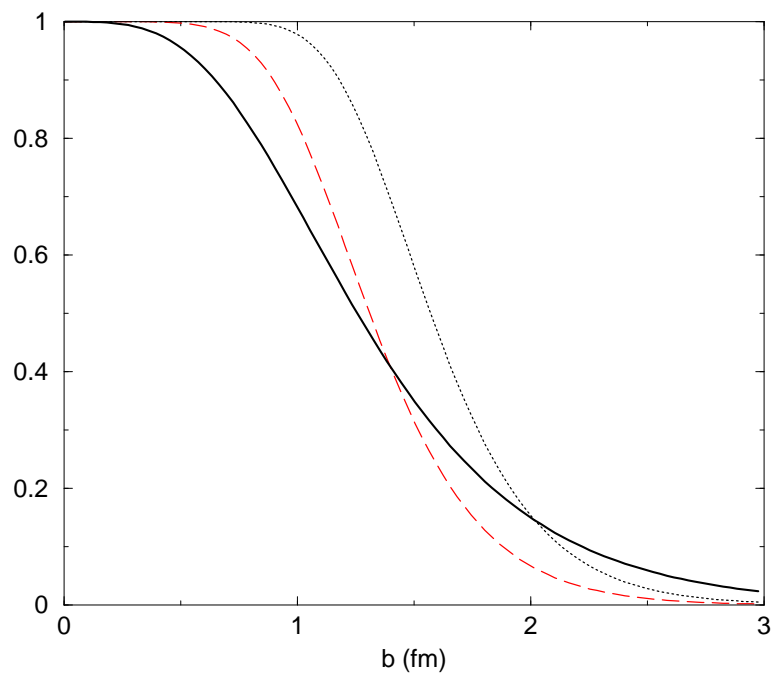

(a)

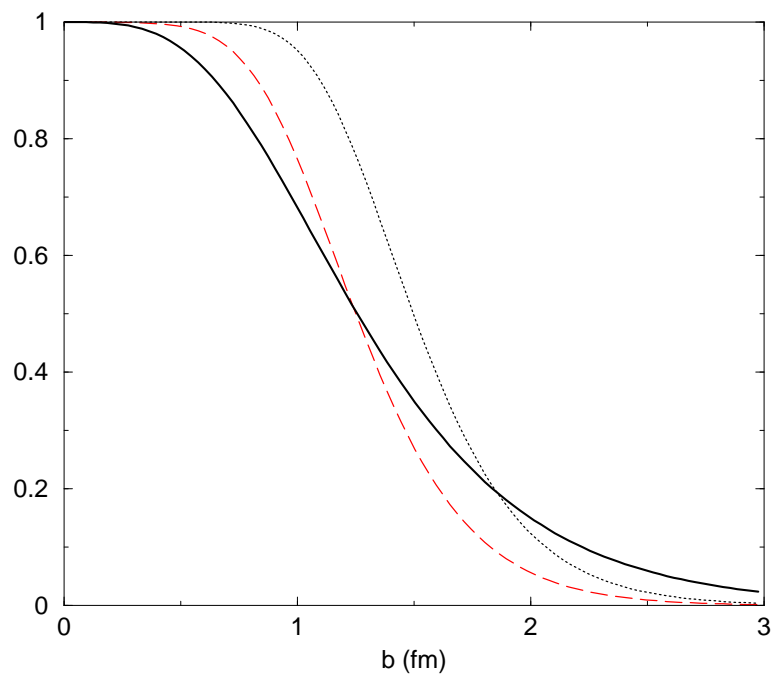

(c)

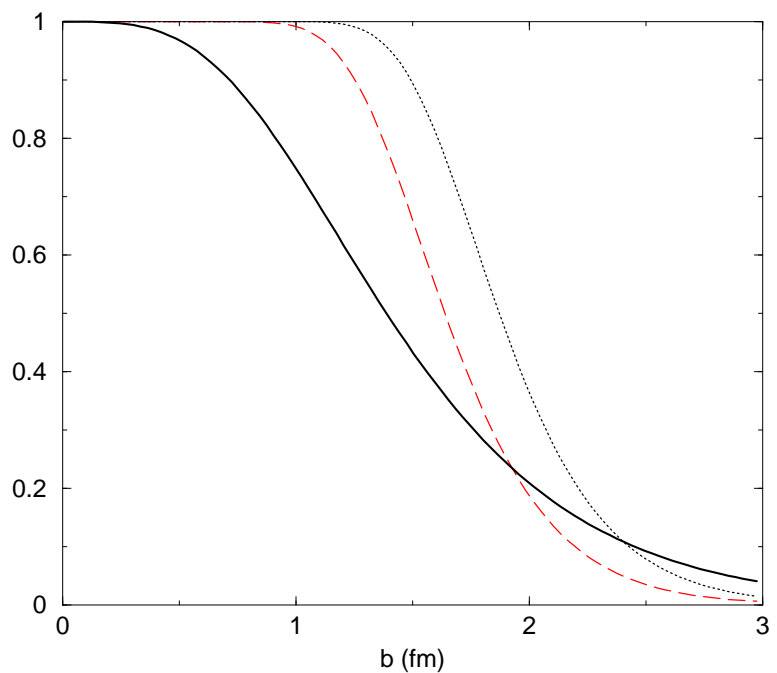

(b)

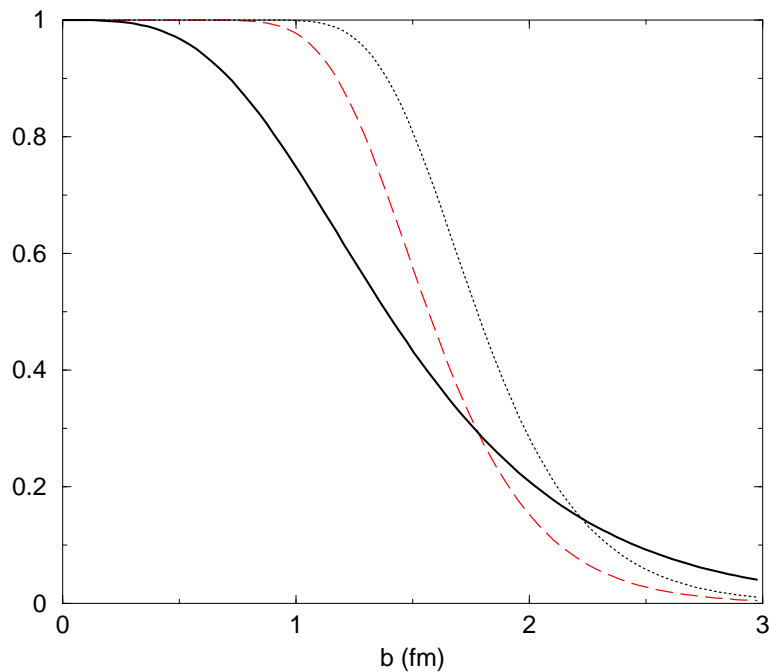

(d)

FIG. 3: In all of these curves, the solid black curve is the extrapolation of the fit of Islam et al. (keeping only the diffractive, assymtotic limit). The black dotted curve is the calculation with $p_{t}=2.5 \mathrm{GeV}$ and the red-dashed curve is for $p_{t}=3.5 \mathrm{GeV}$. The upper plots are calculated using CTEQ6M parton distributions and the lower plots are calculated used MRST parton distributions. (a,c) $\sqrt{s}=14 \mathrm{TeV}$. (b,d) $\sqrt{s}=50 \mathrm{TeV}$. These calculations are done assuming identical partons - Eq. (26).

case: the lower red-dashed curve is with $p_{t}=3.5 \mathrm{GeV}$, and the upper dotted curve is with $p_{t}=2.5 \mathrm{GeV}$. In both of these calculations, we have used CTEQ6M gluon distribution functions. For comparison, we have shown the profile for the total inelastic cross section obtained from the fits of Islam et al 22] (It is difficult at present to estimate the uncertainties in the extrapolation of models for the inelastic impact parameter profile to the LHC energy. However the first data from LHC on elastic $p p$ scattering will essentially eliminate this uncertainty.). In Fig. $3 \mathrm{a}$, the calculation with a cutoff at $p_{t}=2.5 \mathrm{GeV}$ results in a value for $\Gamma_{j e t s}^{\text {inel }}$ that is larger than the inelastic profile function, $\Gamma^{\text {inel }}$ at $b \gtrsim 1.5 \mathrm{fm}$, whereas the cutoff of $p_{t}^{c}=3.5 \mathrm{GeV}$ leads to a violation only at small values of $b$ where the uncorrelated approximation likely breaks down. Therefore, the $p_{t}=2.5 \mathrm{GeV}$ cutoff is certainly inappropriate for the maximum LHC energies. In Fig. $3 \mathrm{~b}$ we show the calculation for the higher energy $\sqrt{s}=50 \mathrm{TeV}$. Here we see that the $p_{t}$ needs to be pushed even higher than $3.5 \mathrm{GeV}$ to avoid a contradiction with unitarity. To test the sensitivity to the type of parton distribution being used, we have repreated the calculation using MRST parton distributions in plots $(\mathrm{c}, \mathrm{d})$. We see that the above conclusions are essentially unchanged.

It is worth emphasizing that typical values of $b$ for jet production are much smaller, $\sim 0.8 \mathrm{fm}$ than the ones for which we obtained the constraint. Since the typical gluon densities involved for $b \sim 0.8 \mathrm{fm}$ are significantly 
larger (of the order of factor 3, compare Eq. (14)) than for $b \sim 1.5 \mathrm{fm}$, the taming mechanism should be effective in the much broader range of $p_{t}$.

This result supports the cutoffs used in DPMjet II and III [3] in which the cutoff at this energy scale is roughly $6 \mathrm{GeV}$, and SIBYLL 2.1 [6] in which the cutoff is roughly $5 \mathrm{GeV}$ independent of $b$. However, in our logic it seems natural for the cutoff to inrease with a decrease of $b$.

Our results disagree with the use of a low fixed cutoff as in QGSjet 5] where the minimum $p_{t}$ is fixed at $1.5 \mathrm{GeV}$, and neXus [4] where the cutoff is fixed at $2.0 \mathrm{GeV}$. Note that it is to natural to expect that the $p_{t}^{2}$ cutoff is proportional to average gluon density encountered for a given impact parameter. Hence our limit for $b \sim 1.5 \mathrm{fm}$ corresponds to a much larger value of the cutoff for more typical $b$-values for the hard collisions - say production of two jets where average $b$ are $\sim 1 \mathrm{fm}$.

We have also repeated the calculation using the assumption of non-identical partons - Eq. (25). This is shown in Fig. 4. We see that our above conclusions regarding the minimum $p_{t}$ are not substantially affected.

One source of uncertainty is in the true shape of the $t$-distribution of the two-gluon form factor. To test the sensitivity to this, we have repeated the analysis of the previous section using the more commonly used Gaussian model for the $t$-dependence of $P_{2}$ rather than the twogluon form factor. We fix the slope by demanding that $\left\langle b^{2}\right\rangle$ for the Gaussian parameter equal the value of $\left\langle b^{2}\right\rangle$ determined from the two-gluon form factor. The result is shown in Fig. 5 In this case, it seems that using the Gaussian parameterization leads to a greater violation of unitarity than the GPD.

\section{INTERACTION RADIUS AT VERY HIGH ENERGIES}

In this section we give a more general discussion of semi-hard interaction radius. We argue that it should be at least as large as what is used in the previous section to maintain consistency with DIS. A common approach to modeling the impact parameter dependence in extrapolations to very high energies is to write the profile function as,

$$
\Gamma(s, b)=1-\exp [-\chi(s, b)] .
$$

Here $\chi(s, b)$ is assumed to describe the phase shift produced by each scattering of the proton constituents.

In extrapolations to ultra-high energies it is also assumed that the basic parton-parton profile function can be decomposed into the sum of a term for soft scattering and for hard scattering:

$$
\Gamma(s, b)=1-\exp \left\{-\chi_{h}(s, b)-\chi_{s}(s, b)\right\} .
$$

Corrections to this simple model are taken into account in more sophisticated versions of the eikonal model that include, for example, diffraction and triple Pomeron exchange [], though for the remaining discussions of this section, it will be sufficient to use Eq. (28). The soft part $\chi_{s}(s, b)$ is modeled phenomenologically, whereas the semi-hard term $\chi_{h}(s, b)$ can be calculated with the aid of Eq. (11). However, we stress that since each term in the exponent of Eq. (27) is quite model dependent the relationship between the unitarity of the total cross section and the large size of the semi-hard contribution, $\chi_{h}(s, b)$ is unclear, and is likely to depend strongly on how we model the soft parts, etc. One can extract constraints on the UHE cross section indirectly by noting that the inclusive cross sections are obtained via sums over exclusive $2 n$ minijet cross sections,

$$
\sigma_{t o t}=\sum_{n=0}^{\infty} \tilde{\sigma}_{2 n}, \quad \sigma_{2 j e t}^{i n c}=\sum_{n=1}^{\infty} n \tilde{\sigma}_{2 n} .
$$

(Here it is assumed that the total cross section is dominated by jet pairs.) As in the previous section, we use a tilde on exclusive quantities. The total and elastic cross sections are, from Eqs. (4) and (5),

$$
\begin{aligned}
\sigma_{\text {tot }} & =2 \int d^{2} \mathbf{b}\left[1-\exp \left\{-\chi_{h}(b)-\chi_{s}(b)\right\}\right], \\
\sigma_{\text {ela }} & =\int d^{2} \mathbf{b}\left[1-\exp \left\{-\chi_{h}(b)-\chi_{s}(b)\right\}\right]^{2} .
\end{aligned}
$$

In [10], for example, a minimum value for $p_{t}^{c}$ was determined by requiring fits of $\sigma_{\text {tot }}$ and $\sigma_{\text {ela }}$ to data for the elastic cross section to be consistent with Eqs. (29). In the semi-hard eikonal factor, a simple Gaussian model is used,

$$
\chi_{h}(b)=\frac{\sigma_{h}(s)}{8 \pi R_{0}^{2}} \exp \left\{-b^{2} / 4 R_{0}^{2}\right\} .
$$

$R_{0}$ is the interaction radius in impact parameter space at the reference energy scale. (In general, there should also be a non-zero Regge slope $\alpha^{\prime}$ leading to diffusion in the transverse plane as energy is increased.)

\section{A. Comparison with Deeply Inelastic Scattering}

From Eq. (IIIB) and Eq. (32) we can extract the equivalent of Eq. (17) for the Gaussian model,

$$
P_{2}^{\text {gauss }}\left(b, s, p_{t}^{c}\right)=\frac{1}{4 \pi R_{0}^{2}} \exp \left\{-b^{2} / 4 R_{0}^{2}\right\} .
$$

When fitted to data the two over-lap functions Eq. (33) and Eq. (17) should be in rough agreement. In particular, both models should yield similar numerical values for $\left\langle b^{2}\right\rangle$ calculated with Eq. (18). For example, if Eq. (32) is used with a radius, $R_{0}=3.5 \mathrm{GeV}^{-2}$ and a transverse momentum cutoff of $p_{t}^{c}=3.5 \mathrm{GeV}$, one obtains a mean impact parameter $\sqrt{\left\langle b^{2}\right\rangle}$ of $0.75 \mathrm{fm}$, whereas if Eq. (17) is used one obtains $\sqrt{\left\langle b^{2}\right\rangle}=0.87 \mathrm{fm}$. In this case there is at least rough agreement between the two models. 


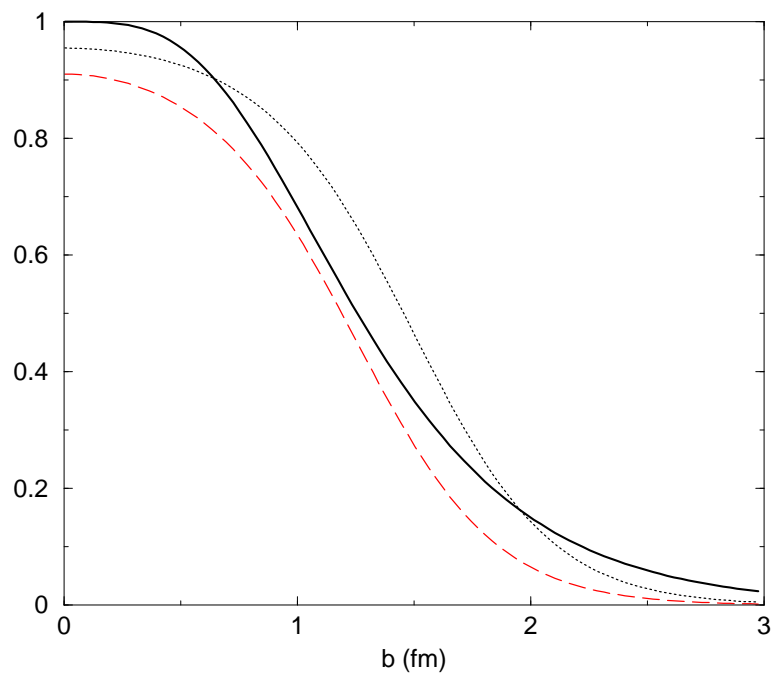

(a)

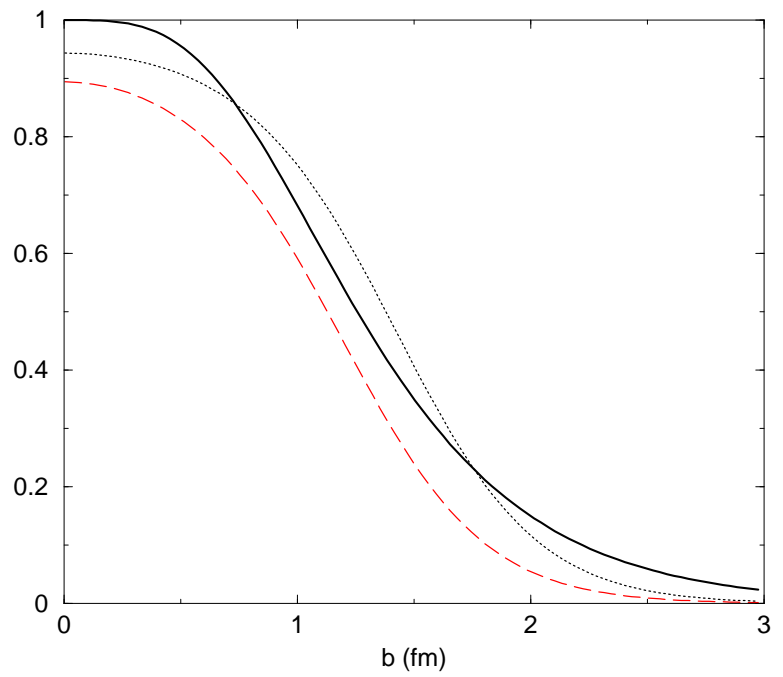

(c)

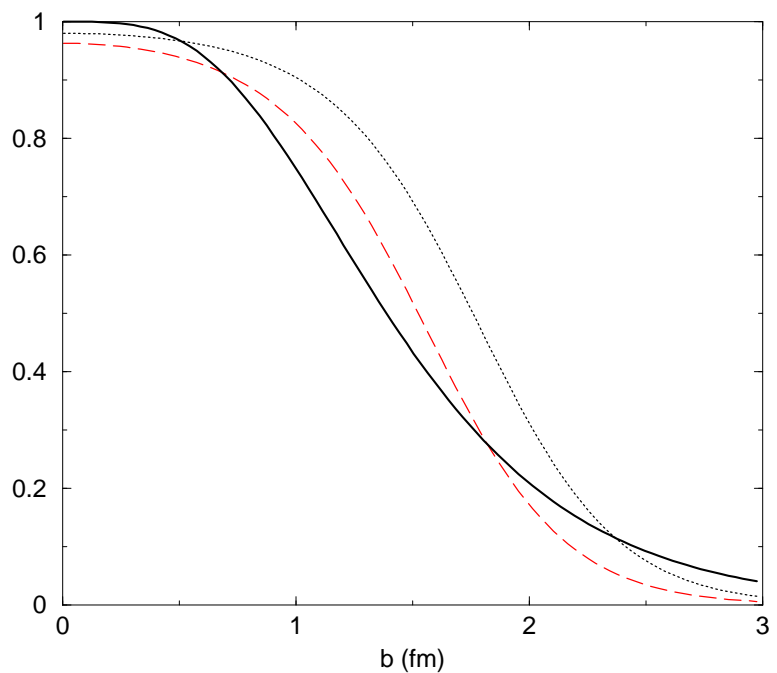

(b)

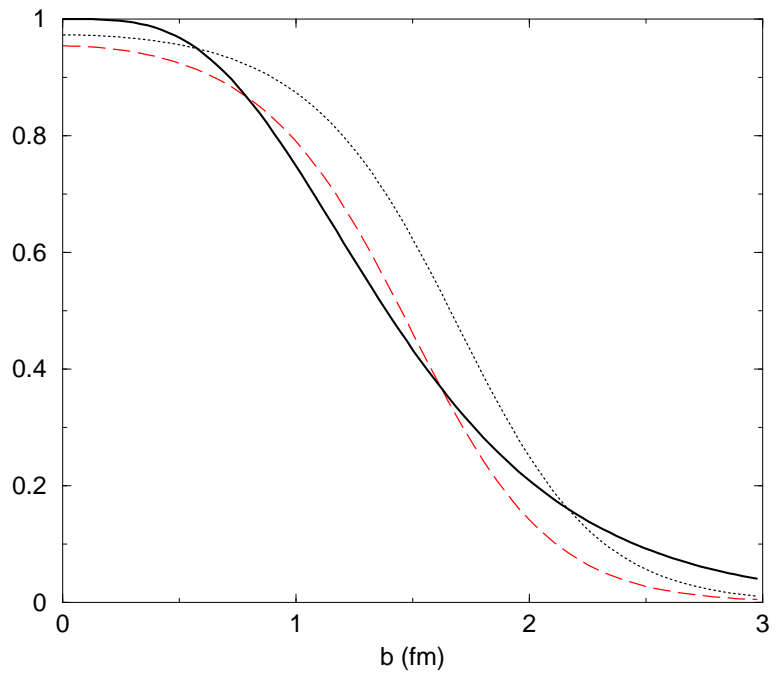

(d)

FIG. 4: In all of these curves, the solid black curve is the extrapolation of the fit of Islam et al. (keeping only the diffractive, asymptotic limit). The black dotted curve is the calculation with $p_{t}=2.5 \mathrm{GeV}$ and the red-dashed curve is for $p_{t}=3.5 \mathrm{GeV}$. The upper plots are calculated using CTEQ6M parton distributions and the lower plots are calculated used MRST parton distributions. Furthermore, the calculation is done assuming non-identical partons - Eq. (25). $(\mathrm{a}, \mathrm{c}) \sqrt{s}=14 \mathrm{TeV}$. $(\mathrm{b}, \mathrm{d}) \sqrt{s}=$ $50 \mathrm{TeV}$.

In Ref. [10] it is found that a fit to the total cross section is also possible with a radius of $R_{0}=1.5 \mathrm{GeV}^{-2}$ and $p_{t}^{c}=2.5 \mathrm{GeV}$. However, in this case Eq. (32) produces a mean impact parameter equal to $\sqrt{\left\langle b^{2}\right\rangle}$ of $0.48 \mathrm{fm}$, whereas using Eq. (17) produces $\sqrt{\left\langle b^{2}\right\rangle}=0.89$ fm. For this scenario, there is clearly an inconsistency between the Gaussian model and the impact parameter dependence extracted from DIS. The spread in impact parameter space for these two scenarios is shown in Fig. 6.

We can gain further insight by examining how the width of the impact parameter dependence of $\mathcal{F}_{g}(x, \rho, \mu)$ relates to the total $q \bar{q}$-proton cross section. In DIS at high energy (or low- $x$ ), cross sections are usually written in the target rest frame in term of the cross section for the interaction of a $q \bar{q}$ dipole with the target proton. The $q \bar{q}$ cross section is,

$$
\sigma_{\text {tot }}^{q \bar{q}}(d, x)=\frac{\pi^{2}}{3} d^{2} \alpha_{s}\left(\lambda / d^{2}\right) x g\left(x, \lambda / d^{2}\right),
$$

where $d$ is the transverse size of the $q \bar{q}$ pair, and $x$ is the longitudinal momentum fraction carried by the struck gluon [25]. Given the total cross section for the $q \bar{q}$-proton interaction and the GPD in Eq. (13), we can write the amplitude for dipole scattering as,

$$
A_{q \bar{q}}(x, t)=i \hat{s} \sigma_{\text {tot }}^{q \bar{q}}(d, x) F_{g}(x, t, \mu),
$$




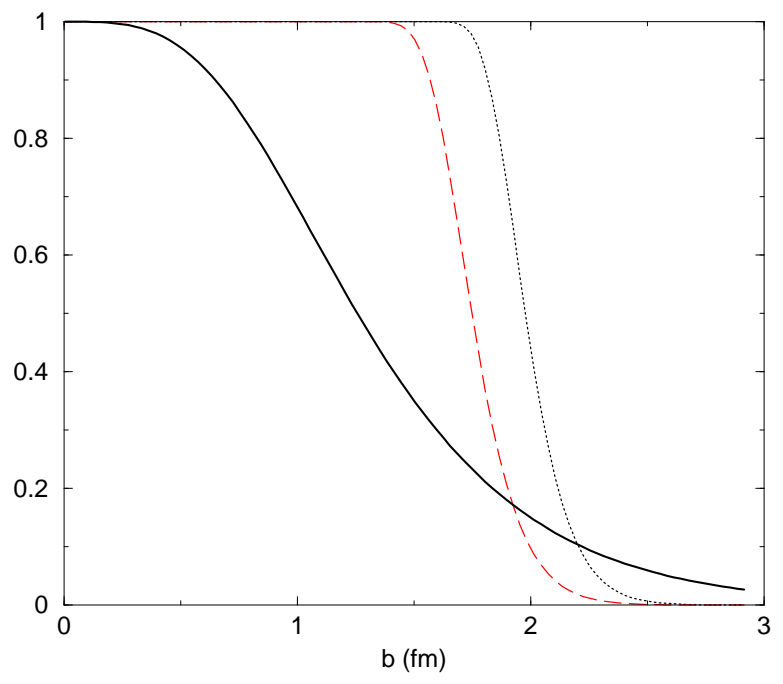

(a)

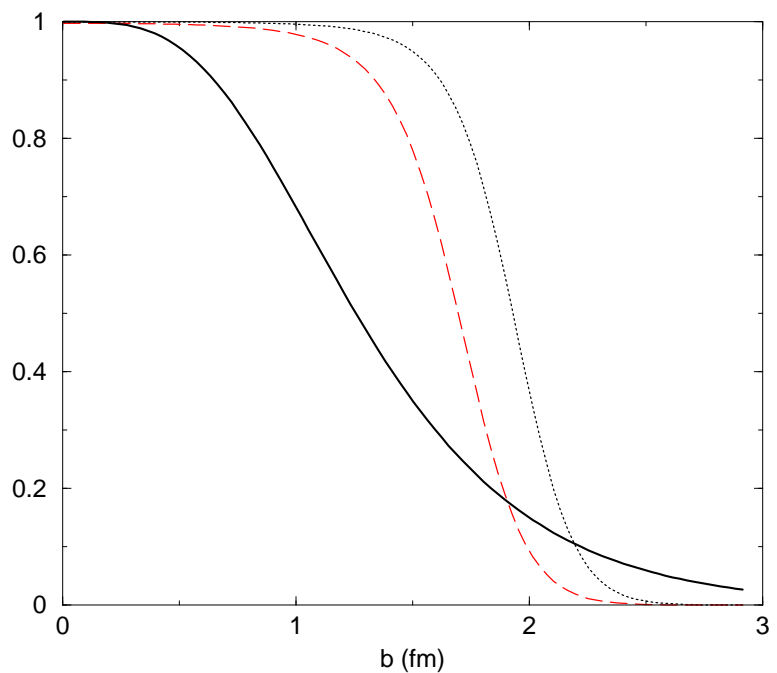

(b)

FIG. 5: For these curves we use a Gaussian model for $P_{2}$. Here $\sqrt{s}=14 \mathrm{TeV}$ and we use CTEQ6M parton distributions. (a) Calculation for identical partons. (b) For non-identical partons.
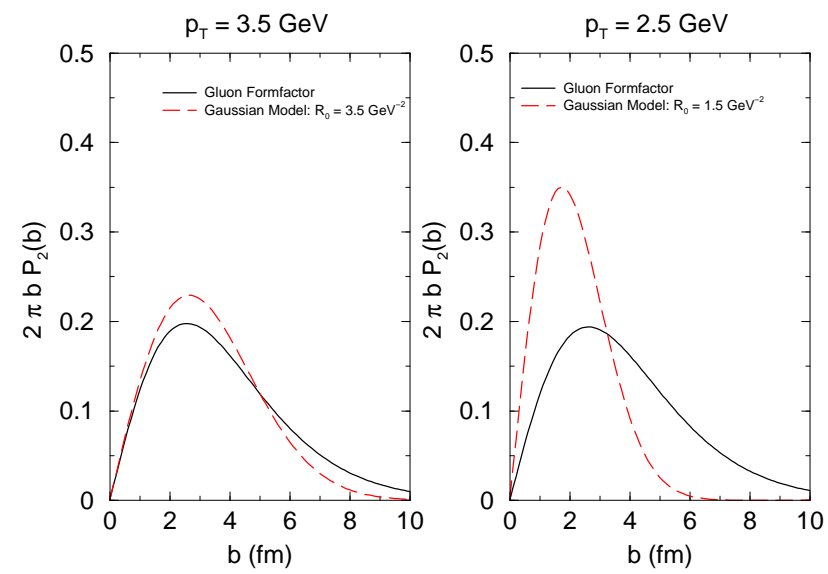

FIG. 6: A comparison of the distribution in impact parameter space for minijet production as predicted by the Gaussian model, Eq. (33) and the 2-gluon form factor, Eq. (17).

Since we are in the high energy limit, we neglect a small real part. $\sqrt{\hat{s}}$ is the center-of-mass energy of the dipoleproton collins. We then invert Eq. 35] to obtain a profile function for the $q \bar{q}$-proton cross section for a pair of size $d$ and for a gluon momentum fraction, $x$ :

$$
\begin{aligned}
& \Gamma_{h}^{q \bar{q}}(b, x)= \\
& \frac{\sigma_{t o t}^{q \bar{q}}(d, x) m_{g}^{2}(x, \mu)}{4 \pi}\left(\frac{m_{g}(x, \mu) b}{2}\right) K_{1}\left(m_{g}(x, \mu) b\right) .
\end{aligned}
$$

In Eq. (2), the hard scale at which the gluon density and the strong coupling are evaluated is $p_{t}$ whereas in the DIS expression, Eq. (34), it is related to the inverse size of the dipole's transverse size, $\lambda / d^{2}$, where $\lambda$ is between
4 and $10[16$. Because of the universality of the GPD, the gluon density in Eq. (34) should be the same as that appearing in Eq. (2) so long as we consider a dipole size $d$ such that $\lambda / d^{2} \sim p_{t}^{2}$. We are interested in the region of the integral close to $p_{t}^{c}$ and for typical values of $x \approx \bar{x}$.

Therefore, we evaluate Eq. (36) at $d_{c}=\sqrt{\lambda /\left(p_{t}^{c}\right)^{2}}$. Using the FSW expression provided in Appendix B for $m_{g}\left(\bar{x}, p_{t}^{c}\right)$ yields the solid curve shown in Fig. 7. The profile function calculated this way is well within the bounds of the unitarity constraint which is consistent with observations at HERA.

However, for $\left\langle b^{2}\right\rangle$ to agree with the prediction from the Gaussian model with $R_{0}=1.5 \mathrm{GeV}^{-2}$ and $p_{t}^{c}=2.5 \mathrm{GeV}$, we find that we must increase the value of $m_{g}$ by about a factor of 1.9. This leads to the dashed profile function shown in Fig. 7 which exceeds the unitarity bound at small values of $b$. From Fig. 6] we see that, for the DIS calculation to remain consistent with unitarity, the $p_{t}$ cutoff in the hadron-hadron jet cross section Eq. (2) should actually be pushed higher as the profile for hard partons becomes narrower. In other words, a narrow profile for hard scattering leads to a more rapid approach to saturation-like physics in the DIS calculation. To avoid a conflict with the unitarity in DIS, and to avoid a contradiction with the observation at HERA that the amplitude for the dipole scattering is relatively far from the black disk limit even when $x=10^{-4} \sim 10^{-3}$ (at least unless the scales are greater than $\sim 2 \mathrm{GeV}^{2}$, see [15]) we must use a radius-squared for the hard interaction that is greater than $\sim 3.5 \mathrm{GeV}^{-2}$. 


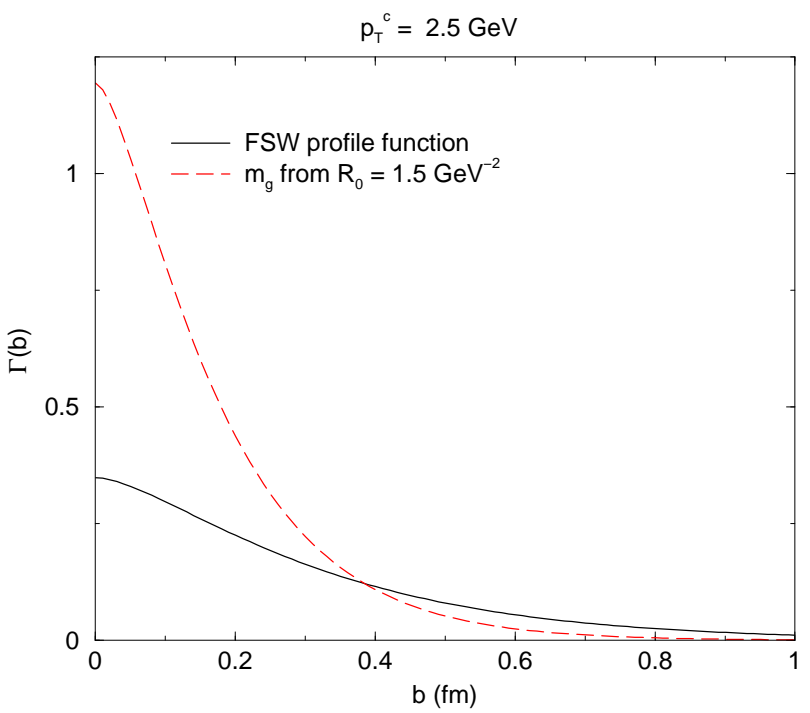

FIG. 7: The profile function (solid curve) calculated using Eq. 36] To match with the Gaussian model, $m_{g}$ must be increased by a factor of 1.9 - resulting in the dashed curve.

\section{CONCLUSIONS}

In this paper we have analyzed the region of applicability of the pQCD formula for the minijet production in hadron-hadron collisions. Based on the unitarity arguments in impact parameter space we have set a constraint on the minimum value of $p_{t}$ for which the formula can be used. The model satisfies the unitarity by construction, i.e. by the summation of the multiple scatterings, without any correlations in impact parameter space. For the LHC-scale energy $\sqrt{s}=14 \mathrm{TeV}$ the minimum value of $p_{t}^{c} \gtrsim 2.5 \mathrm{GeV}$ and for the cosmic ray energies for $p p$ collisions $\sqrt{s}=50 \mathrm{TeV}, p_{t} \gtrsim 3.5 \mathrm{GeV}$.

We again stress that these values are for large impact parameters $\sim 1.5 \mathrm{fm}$. Though our simple analysis is not effective at small impact parameters due to the neglect of correlations and due to $\Gamma(s, b) \sim 1$, it is reasonable to expect the minimum $p_{t}^{c}$ to rise as the gluon density rises. Thus, the true minimum $p_{t}^{c}$ is probably even larger than what we find here.

For the case of the cosmic ray interaction of protons with air, the typical gluon densities are at least factor five larger than the gluon densities encountered in the $p p$ case at $b \sim 1.5 \mathrm{fm}$.

Note that the values of the minimum $p_{t}$ are much larger than any conceivable soft scale. Therefore, the dynamical mechanism for violation of the leading twist formalism which we find here must include some kind of non-linear strong field effects at relatively small coupling.

The analysis can be extended for a more sophisticated model which includes the correlations for multi-jet production in the impact parameter space.

We have also analyzed in detail the dominant regions of the integrand in the minijet formula and shown that the dominant configurations for small energies are when the jets are produced with approximately equal but opposite rapidities. When the energy is increased the configurations with the jets going into the same rapidity hemisphere are equally important. This includes the forward regime in hadron scattering where the saturation corrections in the gluon density are likely to be important at the LHC energy.

\section{Acknowledgments}

We thank Ralf Engel, Leonid Frankfurt and Daniele Treleani for discussions. We also thank Werner Vogelsang for calculations of the $K$ factor in jet production. This research has been supported by the U.S. D.O.E. under grants number DE-FG02-90ER-40577, DE-FGO293ER-40771 and by the Polish Committee for Scientific Research grant No. KBN 1 P03B 02828.

\section{Appendix A}

\section{Parametrization of the mass parameter $m_{g}$ in the profile function}

The mass parameter $m_{g}$ in (13) is related to the inverse of the average impact parameter corresponding to the profile distribution (14)

$$
\left\langle\rho^{2}\right\rangle=\frac{8}{m_{g}^{2}} .
$$

In [21] the following parametrization was found for the $x$ and scale dependence of $m_{g}$

$$
\left\langle\rho^{2}\right\rangle\left(x, Q_{0}^{2}\right)=\max \left\{0.31 \mathrm{fm}^{2}+0.0194 \mathrm{fm}^{2} \ln \frac{0.1}{x}, 0.28 \mathrm{fm}^{2}\right\},
$$

and

$$
\left\langle\rho^{2}\right\rangle\left(x, Q^{2}\right)=\left\langle\rho^{2}\right\rangle\left(x, Q_{0}^{2}\right)\left(1+A \ln \frac{Q^{2}}{Q_{0}^{2}}\right)^{-a}
$$

where

$$
Q_{0}^{2}=3 \mathrm{GeV}^{2}, \quad A=1.5, \quad a=0.0090 \ln \frac{1}{x} .
$$

[1] T. Sjostrand, S. Mrenna and P. Skands, JHEP 0605, 026 (2006) arXiv:hep-ph/0603175.
[2] M. Bahr et al., arXiv:0711.3137 [hep-ph]. 
[3] J. Ranft, Phys. Rev. D 51, 64 (1995).

S. Roesler, R. Engel and J. Ranft: (hep-ph/0012252), in Proc. of Int. Conf. on Advanced Monte Carlo for Radiation Physics, Particle Transport Simulation and Applications (MC2000), Lisbon, Portugal, 23-26 Oct. 2000, A. Kling, F. Barao, M. Nakagawa, L. Tavora, P. Vax eds., Springer-Verlag Berlin, 2000

S. Roesler, R. Engel and J. Ranft: Proc. of 27th ICRC (Hamburg),p.439,2001

[4] H. J. Drescher, M. Hladik, S. Ostapchenko, T. Pierog and K. Werner, Phys. Rept. 350, 93 (2001) arXiv:hep-ph/0007198.

S. S. Ostapchenko, T. Pierog and K. Werner, ICRC (Hamburg), p.446,2001

[5] N. N. Kalmykov, S. S. Ostapchenko and A. I. Pavlov, Nucl. Phys. Proc. Suppl. 52B, 17 (1997).

S. J. Sciutto, J. Knapp and D. Heck,

[6] J. Engel, T. K. Gaisser, T. Stanev and P. Lipari, Phys. Rev. D 46, 5013 (1992).

R. S. Fletcher, T. K. Gaisser, P. Lipari and T. Stanev, Phys. Rev. D 50, 5710 (1994).

[7] A. Capella, J. Tran Thanh Van and J. Kwieciński, Phys. Rev. Lett. 58, 2015 (1987).

P. Aurenche, F. W. Bopp, A. Capella, J. Kwieciński, M. Maire, J. Ranft and J. Tran Thanh Van, Phys. Rev. D 45, 92 (1992).

[8] R. Engel, Nucl. Phys. Proc. Suppl. 122, 40 (2003).

[9] L. Ametller and D. Treleani, Int. J. Mod. Phys. A 3, 521 (1988).

[10] R. Engel, T. K. Gaisser and T. Stanev, Prepared for 27th International Cosmic Ray Conference (ICRC 2001), Hamburg, Germany, 7-15 Aug 2001

[11] L. V. Gribov, E. M. Levin and M. G. Ryskin, Phys. Rept. 100, 1 (1983).

[12] E. Iancu and R. Venugopalan, arXiv:hep-ph/0303204.
[13] K. J. Golec-Biernat and M. Wusthoff, Phys. Rev. D 59, 014017 (1999) arXiv:hep-ph/9807513.

[14] K. J. Golec-Biernat and M. Wusthoff, Phys. Rev. D 60, 114023 (1999) arXiv:hep-ph/9903358.

[15] S. Munier, A. M. Stasto and A. H. Mueller, Nucl. Phys. B 603, 427 (2001) arXiv:hep-ph/0102291.

[16] M. McDermott, L. Frankfurt, V. Guzey and M. Strikman, Eur. Phys. J. C 16, 641 (2000) arXiv:hep-ph/9912547.

T. Rogers, V. Guzey, M. Strikman and X. Zu, Phys. Rev. D 69, 074011 (2004) arXiv:hep-ph/0309099.

[17] H. Kowalski and D. Teaney, Phys. Rev. D 68, 114005 (2003) arXiv:hep-ph/0304189.

[18] J. Pumplin, D. R. Stump, J. Huston, H. L. Lai, P. Nadolsky and W. K. Tung, JHEP 0207, 012 (2002) arXiv:hep-ph/0201195.

[19] L. Frankfurt, M. Strikman and C. Weiss, Ann. Rev. Nucl. Part. Sci. 55, 403 (2005) arXiv:hep-ph/0507286. F. Gelis, A. M. Stasto and R. Venugopalan, Eur. Phys. J. C 48, 489 (2006) arXiv:hep-ph/0605087.

[20] L. Frankfurt and M. Strikman, Phys. Rev. D 66, 031502 (2002) arXiv:hep-ph/0205223.

[21] L. Frankfurt, M. Strikman and C. Weiss, Phys. Rev. D 69, 114010 (2004) arXiv:hep-ph/0311231.

[22] M. M. Islam, R. J. Luddy and A. V. Prokudin, Mod. Phys. Lett. A 18, 743 (2003) arXiv:hep-ph/0210437.

[23] G. Calucci and D. Treleani, Phys. Rev. D 60, 054023 (1999) arXiv:hep-ph/9902479.

[24] Note that, usually saturation line is referred to as line in $(x, \mu)$ space where $\mu$ is the typical scale. Here, even though the scale $p_{t}$ is fixed in the contour plots we still have the line, since $x_{1,2}$ are functions of rapidities $y_{1}, y_{2}$.

[25] This formula is derived for small $d$ when $\sigma_{\text {tot }} \approx \sigma_{\text {inel }}$ 